\title{
Research Square \\ Exact Density Matrix Elements for a Driven Dissipative Bosonic Mode in a Kerr-Like Medium
}

\section{Sh. Saedi}

University of Kurdistan

F. Kheirandish ( $\sim$ f.kheirandish@uok.ac.ir)

University of Kurdistan

\section{Research Article}

Keywords: matrix elements, density matrix, bosonic mode

Posted Date: May 19th, 2021

DOl: https://doi.org/10.21203/rs.3.rs-513805/v1

License: (9) This work is licensed under a Creative Commons Attribution 4.0 International License. Read Full License 


\title{
Exact density matrix elements for a driven dissipative bosonic mode in a Kerr-like medium
}

\author{
Sh. Saedi $^{1}$ and F. Kheirandish*1 \\ ${ }^{1}$ Department of Physics, University of Kurdistan, P.O.Box 66177-15175, Sanandaj, Iran
}

(Dated: May 18, 2021)

\begin{abstract}
For a prototype Hamiltonian describing a driven, dissipative bosonic mode in a Kerr-like medium, the exact matrix elements of the reduced density matrix are obtained from a generating function in terms of the normal characteristic functions. The approach is based on the Heisenberg equations and operator calculus. The special and limiting cases are discussed.
\end{abstract}




\section{INTRODUCTION}

Experimental methods in the design of devices used in nanoscale physics and quantum technologies have advanced much in recent years and have led to very high accuracy in measuring instruments. These devices are very sensitive to external potentials and types of noise because their operation is in the domain of quantum mechanics. Therefore, understanding the performance and quantum dynamics of these devices is essential to control them and correct unwanted behaviors. A real quantum device is not an isolated system and interacts with its environment or there may be some external classical sources applied to the system. Our favorite quantum devices belong to a much wider class of quantum systems, nowadays referred to as open quantum systems [1]. The subject of open quantum systems (systems that exchange information with their surroundings) covers a vast range of applications in quantum physics and other related subjects. Generally, by an open quantum system, we mean a possibly driven system as the main system interacting with some other systems modeling its environment. In the terminology of open quantum systems, the main system together with its environment under the influence of external classical sources is considered as a closed system. Therefore, the time-evolution of the total system can be described by a total density matrix evolving unitarily. If we are interested in the dynamics of the main system or any other subsystem in the environment, the other degrees of freedom should be traced out to get the favorite reduced density matrix. The quantum Brownian motion is an example of an open quantum system that is extensively studied in various branches of physics [2-9]. Another important feature of nanoscale quantum devices is their thermodynamical properties. Usually, the quantum fluctuations of the physical quantities in nanoscale quantum devices are of the same order of magnitude as their expectation values leading to a reformulation of thermodynamics in the quantum regime referred to as quantum thermodynamics [10-19]. There are some other quantum systems whose Hamiltonians resemble the Hamiltonian of the quantum Brownian motion in external sources. Here, we have considered the Hamiltonian Eq. (1) which is a general bosonic Hamiltonian describing a bosonic mode interacting with a bosonic bath in a Kerr medium under the influence of external sources. In the framework of Heisenberg equations, we will find exact closed-form expressions for the matrix elements of the reduced density matrix of the main bosonic mode. Knowing the matrix elements of the reduced density matrix, a full investigation of the dynamics of the main subsystem system can be achieved.

\section{THE MAIN DEFINITIONS}

The prototype Hamiltonian that we have considered in this section is the Hamiltonian of a bosonic mode or a quantum harmonic oscillator interacting with its environment under an external classical source in a Kerr like medium. The Hamiltonian is given by

$$
\hat{H}=\overbrace{\hbar \omega_{0} \hat{a}^{\dagger} \hat{a}}^{\hat{H}_{S}}+\overbrace{\sum_{j} \hbar \omega_{j} \hat{b}^{\dagger}{ }_{j} \hat{b}_{j}}^{\hat{H}_{R}}+\overbrace{\sum_{j}\left[\hbar f_{j} \hat{a}^{\dagger} \hat{b}_{j}+\hbar \bar{f}_{j} \hat{b}_{j}^{\dagger} \hat{a}\right]}^{\hat{H}_{S R}}+\overbrace{\hbar k(t) \hat{a}^{\dagger}+\hbar \bar{k}(t) \hat{a}+\hbar \bar{\phi} \hat{a}^{2}+\hbar \phi\left(\hat{a}^{\dagger}\right)^{2}}^{\hat{H}_{\text {ext }}},
$$

where $f_{j}$ are the coupling constants that couple the oscillator to its environment. Here, the complex conjugate of any quantity such as $c$ is denoted by $\bar{c}$ and its norm by $|c|$. The Laplace transform of a function is denoted by $\mu(t)$ with $\tilde{\mu}(s)=\mathcal{L}[\mu(t)]$ with the inverse $\mu(t)=\mathcal{L}^{-1}[\mu(s)]$. In the Hamiltonian Eq. (1) the time-dependent function $k(t)$ represents an external classical force and $\phi$ denotes the coupling between the oscillator and a Kerr like medium. Our goal is to find the exact matrix components of the reduced density matrix of the oscillator as the main system. To do this, we first need to find the temporal evolution of the oscillator ladder operators. By making use of the Heisenberg equations of motion we find (see Supp.Mat-Sec. I)

$$
\begin{aligned}
& \hat{a}(t)=\alpha_{1}(t) \hat{a}(0)-2 i \phi \alpha_{2}(t) \hat{a}^{\dagger}(0)-i \sum_{j} M_{j}(t) \hat{b}_{j}(0)-i \sum_{j}(2 i \phi) N_{j}(t) \hat{b}_{j}^{\dagger}(0)-i \zeta_{1}(t)-i(2 i \phi) \zeta_{2}(t), \\
& \hat{a}^{\dagger}(t)=\bar{\alpha}_{1}(t) \hat{a}^{\dagger}(0)+2 i \bar{\phi} \alpha_{2}(t) \hat{a}(0)+i \sum_{j} \bar{M}_{j}(t) \hat{b}_{j}^{\dagger}(0)-i \sum_{j}(2 i \phi) \bar{N}_{j}(t) \hat{b}_{j}(0)+i \bar{\zeta}_{1}(t)-i(2 i \phi) \bar{\zeta}_{2}(t),
\end{aligned}
$$


where we have defined

$$
\begin{aligned}
\alpha_{1}(t) & =\mathcal{L}^{-1}\left[\frac{\tilde{\tilde{G}}(s)}{\tilde{L}(s)}\right], \\
\alpha_{2}(t) & =\mathcal{L}^{-1}\left[\frac{1}{\tilde{L}(s)}\right], \\
\tilde{L}(s) & =|\tilde{G}(s)|^{2}-4|\phi|^{2}, \\
\tilde{G}(s) & =s+i \omega_{0}+\tilde{\chi}(s), \\
M_{j}(t) & =f_{j} \int_{0}^{t} d t^{\prime} e^{i \omega_{j}\left(t-t^{\prime}\right)} \alpha_{1}\left(t^{\prime}\right), \\
N_{j}(t) & =\bar{f}_{j} \int_{0}^{t} d t^{\prime} e^{-i \omega_{j}\left(t-t^{\prime}\right)} \alpha_{2}\left(t^{\prime}\right), \\
\zeta_{1}(t) & =\int_{0}^{t} d t^{\prime} \alpha_{1}\left(t-t^{\prime}\right) k\left(t^{\prime}\right), \\
\zeta_{2}(t) & =\int_{0}^{t} d t^{\prime} \alpha_{2}\left(t-t^{\prime}\right) \bar{k}\left(t^{\prime}\right),
\end{aligned}
$$

Similarly, for the environment operators we find (see Supp.Mat-Sec. I)

$$
\begin{aligned}
& \hat{b}_{j}(t)=\sum_{k}\left[\Lambda_{j k}(t) \hat{b}_{k}(0)+\Lambda_{j k}^{\prime}(t) \hat{b}_{k}^{\dagger}(0)-\Gamma_{j k}(t) \hat{a}(0)-\Gamma_{j k}^{\prime}(t) \hat{a}^{\dagger}(0)-\Omega_{j k}(t)\right], \\
& \hat{b}_{j}^{\dagger}(t)=\sum_{k}\left[\bar{\Lambda}_{j k}(t) \hat{b}_{k}^{\dagger}(0)+\bar{\Lambda}_{j k}^{\prime}(t) \hat{b}_{k}(0)-\bar{\Gamma}_{j k}(t) \hat{a}^{\dagger}(0)-\bar{\Gamma}_{j k}^{\prime}(t) \hat{a}(0)-\bar{\Omega}_{j k}(t)\right],
\end{aligned}
$$

where we have defined

$$
\begin{aligned}
& \Lambda_{j k}(t)=e^{-i \omega_{j} t} \delta_{j k}-\bar{f}_{j} \int_{0}^{t} d t^{\prime} e^{i \omega_{j}\left(t-t^{\prime}\right)} M_{k}\left(t^{\prime}\right), \\
& \Lambda_{j k}^{\prime}(t)=-\bar{f}_{j} \int_{0}^{t} d t^{\prime} e^{i \omega_{j}\left(t-t^{\prime}\right)}(2 i \phi) N_{k}\left(t^{\prime}\right), \\
& \Gamma_{j k}(t)=-i \bar{f}_{j} \int_{0}^{t} d t^{\prime} e^{i \omega_{j}\left(t-t^{\prime}\right)} \alpha_{1 k}\left(t^{\prime}\right), \\
& \Gamma_{j k}^{\prime}(t)=-2 \phi \bar{f}_{j} \int_{0}^{t} d t^{\prime} e^{i \omega_{j}\left(t-t^{\prime}\right)} \alpha_{2 k}\left(t^{\prime}\right), \\
& \Omega_{j k}(t)=-\bar{f}_{j} \int_{0}^{t} d t^{\prime} e^{i \omega_{j}\left(t-t^{\prime}\right)}\left(\zeta_{1}\left(t^{\prime}\right)-(2 i \phi) \zeta_{2}\left(t^{\prime}\right)\right) .
\end{aligned}
$$

\section{REDUCED DENSITY MATRIX ELEMENTS}

Let the initial density matrix at $(t=0)$ be a separable state

$$
\hat{\rho}\left(t_{0}=0\right)=\hat{\rho}_{S}\left(t_{0}=0\right) \otimes \hat{\rho}_{R}\left(t_{0}=0\right),
$$

then the density matrix at a later time is given by

$$
\hat{\rho}(t)=\hat{U}(t) \hat{\rho}\left(t_{0}\right) \hat{U}^{\dagger}(t),
$$

and the reduced density matrix of the bosonic mode can be obtained by tracing out the degrees of the freedom of the environment

$$
\hat{\rho}_{S}(t)=\operatorname{Tr}_{R}\{\hat{\rho}(t)\}
$$


We are interested in the matrix elements of the reduced density matrix. We have

$$
\begin{aligned}
\left\langle n\left|\hat{\rho}_{S}(t)\right| m\right\rangle & =\left\langle n\left|\operatorname{Tr}_{R}\left\{\hat{U}(t) \hat{\rho}\left(t_{0}\right) \hat{U}^{\dagger}(t)\right\}\right| m\right\rangle, \\
& =\left(|m\rangle\langle n| \operatorname{Tr}_{R}\left\{\hat{U}(t) \hat{\rho}\left(t_{0}\right) \hat{U}^{\dagger}(t)\right\}\right), \\
& =\operatorname{Tr}\left\{\left(|m\rangle\langle n| \otimes I_{R}\right) \hat{U}(t) \hat{\rho}\left(t_{0}\right) \hat{U}^{\dagger}(t)\right\}, \\
& =\operatorname{Tr}\{\overbrace{\hat{U}^{\dagger}(t)\left(|m\rangle\langle n| \otimes I_{R}\right) \hat{U}(t)}^{\hat{Q}_{n m}} \hat{\rho}\left(t_{0}\right)\},
\end{aligned}
$$

therefore,

$$
\begin{aligned}
\left\langle n\left|\hat{\rho}_{S}(t)\right| m\right\rangle & =\operatorname{Tr}\left\{\hat{Q}_{n m} \hat{\rho}(0)\right\} \\
& =\operatorname{Tr}\left\{\hat{Q}_{n m} \hat{\rho}_{S}(0) \otimes \hat{\rho}_{R}(0)\right\} .
\end{aligned}
$$

In Eq. (11), the operator $I_{R}$ is the identity operator over the environment Hilbert space. The matrix elements $Q_{m n}$ can be written in terms of ladder operators (see Supp.Mat-Sec. II)

$$
\hat{Q}_{n m}=\frac{1}{\sqrt{m ! n !}} \sum_{s=0}^{\infty} \frac{(-1)^{s}}{s !}\left(\hat{a}^{\dagger}(t)\right)^{m+s}(\hat{a}(t))^{n+s}
$$

where

$$
\begin{aligned}
\hat{a}(t) & =\hat{C}(t)-i(\hat{B}(t)+\zeta(t)), \\
\hat{C}(t) & =\alpha_{1}(t) \hat{a}(0)-2 i \phi \alpha_{2}(t) \hat{a}^{\dagger}(0), \\
\hat{B}(t) & =\sum_{j}\left(M_{j}(t) \hat{b}_{j}(0)+2 i \phi N_{j}(t) \hat{b}_{j}^{\dagger}(0)\right), \\
\zeta(t) & =\left(\zeta_{1}(t)+2 i \phi \zeta_{2}(t)\right),
\end{aligned}
$$

and a similar relation can be written for the harmonic oscillator creation operator. By inserting the expressions for $\hat{a}(t)$ and $\hat{a}^{\dagger}(t)$ into Eq. (13), we obtain

$$
\hat{Q}_{n m}=\frac{1}{\sqrt{m ! n !}} \sum_{s=0}^{\infty} \frac{(-1)^{s}}{s !} \operatorname{Tr}\left\{\left(\hat{C}^{\dagger}(t)+i\left(\hat{B}^{\dagger}(t)+\bar{\zeta}(t)\right)\right)^{m+s}(\hat{C}(t)-i(\hat{B}(t)+\zeta(t)))^{n+s}\right\}
$$

Now for a separable initial state we have

$$
\left\langle n\left|\hat{\rho}_{S}(t)\right| m\right\rangle=\frac{(-1)^{n}}{\sqrt{m ! n !}} \sum_{s=0}^{\infty} \frac{1}{s !} \frac{\partial^{m+s}}{\partial \lambda^{m+s}} \frac{\partial^{n+s}}{\partial(\bar{\lambda})^{n+s}} \operatorname{Tr}\left\{e^{\lambda\left(\hat{C}^{\dagger}(t)+i\left(\hat{B}^{\dagger}(t)+\bar{\zeta}(t)\right)\right)} e^{-\bar{\lambda}(\hat{C}(t)-i(\hat{B}(t)+\zeta(t)))} \hat{\rho}(0)\right\}_{\lambda=\bar{\lambda}=0}
$$

From the definitions of operators $\hat{B}$ and $\hat{C}$, we have

$$
[\hat{B}, \hat{C}]=\left[\hat{B}, \hat{C}^{\dagger}\right]=0
$$

so we can write

$$
\begin{aligned}
& \left\langle n\left|\hat{\rho}_{S}(t)\right| m\right\rangle= \\
& \frac{(-1)^{n}}{\sqrt{m ! n !}} \sum_{s=0}^{\infty} \frac{1}{s !} \frac{\partial^{m+s}}{\partial \lambda^{m+s}} \frac{\partial^{n+s}}{\partial(\bar{\lambda})^{n+s}}\left[e^{i \lambda \bar{\zeta}(t)+i \bar{\lambda} \zeta(t) \overbrace{\operatorname{Tr}_{S}\left\{e^{\lambda \hat{C}^{\dagger}(t)} e^{-\bar{\lambda} \hat{C}(t)} \hat{\rho}_{S}(0)\right\}}^{I_{\hat{C}}} \overbrace{\operatorname{Tr}_{R}\left\{e^{i \lambda \hat{B}^{\dagger}(t)} e^{i \bar{\lambda} \hat{B}(t)} \hat{\rho}_{R}(0)\right\}}^{I_{\hat{B}}}]_{\lambda=\bar{\lambda}=0}},\right. \\
& =\frac{(-1)^{n}}{\sqrt{m ! n !}} \frac{\partial^{m}}{\partial \lambda^{m}} \frac{\partial^{n}}{\partial(\bar{\lambda})^{n}} e^{\partial_{\lambda} \partial_{\bar{\lambda}}}\left[e^{i \lambda \bar{\zeta}(t)+i \bar{\lambda} \zeta(t)} I_{\hat{C}} I_{\hat{B}}\right]_{\lambda=\bar{\lambda}=0} \cdot
\end{aligned}
$$


Eq. (18) is a general result giving the components of the reduced density matrix in terms of a generating function. To proceed, let us assume that the initial state of the environment is a thermal state with temperature $T$

$$
\begin{aligned}
\hat{\rho}_{R}(t) & =\frac{1}{Z_{R}} \prod_{j} e^{-\beta \hbar \omega_{j} \hat{b}_{j}^{\dagger} \hat{b}_{j}}, \\
Z_{R} & =\prod_{j} z_{j}, \\
z_{j} & =\operatorname{Tr}_{j}\left\{e^{-\beta \hbar \omega_{j} \hat{b}_{j}^{\dagger} \hat{b}_{j}}\right\},
\end{aligned}
$$

where $\beta=1 / \kappa_{B} T$ and $\kappa_{B}$ is the Boltzmann constant. Also, $T r_{j}$ denotes the trace over the base vectors corresponding to the $j$ th oscillator of the environment. One can obtain $I_{\hat{B}}$ easily as (see Supp.Mat-Sec. III)

$$
\operatorname{Tr}_{R}\left\{e^{i \lambda \hat{B}^{\dagger}(t)} e^{i \bar{\lambda} \hat{B}(t)} \hat{\rho}_{R}(0)\right\}=e^{\vartheta[\lambda, \bar{\lambda}, t]}
$$

where

$$
\begin{aligned}
\vartheta[\lambda, \bar{\lambda}, t] & =\sum_{k}\left[-\lambda \bar{\lambda}\left(\frac{\left|V_{k}(t)\right|^{2}}{e^{\beta \hbar \omega_{k}}-1}+4|\phi|^{2}\left|N_{k}(t)\right|^{2}\right)+i\left(\lambda^{2} \bar{\phi} \bar{N}_{k}(t) \bar{M}_{k}(t)-\bar{\lambda}^{2} \phi N_{k}(t) M_{k}(t)\right)\right] \\
V_{k}(t) & =M_{k}(t)+2 i \bar{\phi} \bar{N}_{k}(t),
\end{aligned}
$$

therefore,

$$
\begin{aligned}
\left\langle n\left|\hat{\rho}_{S}(t)\right| m\right\rangle & =\frac{(-1)^{n}}{\sqrt{m ! n !}} \sum_{s=0}^{\infty} \frac{1}{s !} \frac{\partial^{m+s}}{\partial \lambda^{m+s}} \frac{\partial^{n+s}}{\partial(\bar{\lambda})^{n+s}}\left[e^{i \lambda \bar{\zeta}(t)+i \bar{\lambda} \zeta(t)} e^{\vartheta[\lambda, \bar{\lambda}, t]} \operatorname{Tr}_{S}\left\{e^{\lambda \hat{C}^{\dagger}(t)} e^{-\bar{\lambda} \hat{C}(t)} \hat{\rho}_{S}(0)\right\}\right]_{\lambda=\bar{\lambda}=0} \\
& =\frac{(-1)^{n}}{\sqrt{m ! n !}} \frac{\partial^{m}}{\partial \lambda^{m}} \frac{\partial^{n}}{\partial(\bar{\lambda})^{n}} e^{\partial_{\lambda} \partial_{\bar{\lambda}}}\left[e^{i \lambda \bar{\zeta}(t)+i \bar{\lambda} \zeta(t)} e^{\vartheta[\lambda, \bar{\lambda}, t]} \operatorname{Tr}_{S}\left\{e^{\lambda \hat{C}^{\dagger}(t)} e^{-\bar{\lambda} \hat{C}(t)} \hat{\rho}_{S}(0)\right\}\right]_{\lambda=\bar{\lambda}=0}
\end{aligned}
$$

\section{THE BOSONIC MODE IS INITIALLY PREPARED IN A COHERENT STATE}

As an application of the general formula Eq. (23), let us assume that the initial state of the main system is a coherent state

$$
\hat{\rho}_{S}(0)=|\gamma\rangle\langle\gamma|
$$

in this case, we have (see Supp.Mat-Sec. IV)

$$
\operatorname{Tr}_{S}\left\{e^{\lambda \hat{C}^{\dagger}(t)} e^{-\bar{\lambda} \hat{C}(t)} \hat{\rho}_{S}(0)\right\}=e^{\alpha_{2}(t)\left(i \lambda^{2} \bar{\phi} \bar{\alpha}_{1}(t)-i \bar{\lambda}^{2} \phi \alpha_{1}(t)-4 \lambda \bar{\lambda}|\phi|^{2} \alpha_{2}(t)\right)} e^{\bar{\gamma}\left(\lambda \bar{\alpha}_{1}(t)+2 i \bar{\lambda} \phi \alpha_{2}(t)\right)} e^{-\gamma\left(\bar{\lambda} \alpha_{1}(t)-2 i \lambda \bar{\phi} \alpha_{2}(t)\right)},
$$

and Eq. (23) can be rewritten as

$$
\begin{aligned}
& \left\langle n\left|\hat{\rho}_{S}(t)\right| m\right\rangle=\frac{(-1)^{n}}{\sqrt{m ! n !}} \frac{\partial^{m}}{\partial \lambda^{m}} \frac{\partial^{n}}{\partial(\bar{\lambda})^{n}} e^{\partial_{\lambda} \partial_{\bar{\lambda}}} \\
& {\left[e^{i(\lambda \bar{\zeta}(t)+\bar{\lambda} \zeta(t))} e^{-4 \lambda \bar{\lambda}|\phi|^{2} \alpha_{2}^{2}(t)+i \alpha_{2}(t)\left(\lambda^{2} \bar{\phi} \bar{\alpha}_{1}(t)-\bar{\lambda}^{2} \phi \alpha_{1}(t)\right)} e^{\vartheta[\lambda, \bar{\lambda}, t]} e^{\left(\lambda \bar{\gamma} \bar{\alpha}_{1}(t)-\bar{\lambda} \gamma \alpha_{1}(t)\right)+2 i \alpha_{2}(t)(\bar{\lambda} \bar{\gamma} \phi+\lambda \gamma \bar{\phi})}\right]_{\lambda=\bar{\lambda}=0} .}
\end{aligned}
$$

Therefore, if the initial state of the main system is a coherent state and the initial state of the environment is a Maxwell Boltzmann thermal state then the elements of the reduced density matrix can be obtained from a generating function given by Eq. (26). For the special case $n=m,(\phi=0)$, we have

$$
\left.P_{n}(t)\right|_{\phi=0}=\frac{(-1)^{n}}{n !}\left(\frac{\partial}{\partial \lambda} \frac{\partial}{\partial \bar{\lambda}}\right)^{n} \overbrace{\sum_{s=0}^{\infty} \frac{1}{s !}\left(\frac{\partial}{\partial \lambda} \frac{\partial}{\partial \bar{\lambda}}\right)^{s}}^{e^{\partial_{\lambda} \partial_{\bar{\lambda}}}} I,
$$


where

$$
\begin{aligned}
I & =e^{-\lambda \bar{\lambda} \eta(t)+\lambda \bar{Z}-\bar{\lambda} Z}, \\
\eta(t) & =\sum_{k} \frac{\left|M_{k}(t)\right|^{2}}{e^{\beta \hbar \omega_{k}}-1}, \\
Z & =-i \zeta(t)+\gamma \alpha_{1}(t), \\
\bar{Z} & =i \bar{\zeta}(t)+\bar{\gamma} \bar{\alpha}_{1}(t) .
\end{aligned}
$$

Therefore, (see Supp.Mat-Sec. V)

$$
\left.P_{n}(t)\right|_{\phi=0}=\frac{2 \pi e^{-\frac{|Z|^{2}}{1-\eta(t)}}}{1-\eta(t)}\left(\frac{\eta(t)}{\eta(t)-1}\right)^{n} L_{n}\left(-\frac{|Z|^{2}}{\eta(t)(\eta(t)-1)}\right),
$$

where $L_{n}[x]$ is a Laguerre polynomial of degree $n$. In the absence of a Kerr medium, $(\phi=0)$ and in low temperature regime $(\beta \rightarrow \infty)$, we have

$$
\begin{aligned}
& \eta(t) \rightarrow 0 \\
& V_{k}(t)=M_{k}(t), \\
& \tilde{L}(s)=|\tilde{G}(s)|^{2} \Longrightarrow \alpha_{1}(t)=\mathcal{L}^{-1}\left[\frac{1}{\tilde{G}(s)}\right], \\
& \tilde{G}(s)=s+i \omega_{0}+\tilde{\chi}(s) .
\end{aligned}
$$

Now by making use of the identity

$$
\lim _{\eta \rightarrow 0}\left(\frac{\eta(t)}{\eta(t)-1}\right)^{n} L_{n}\left(-\frac{|Z|^{2}}{\eta(t)(\eta(t)-1)}\right)=\frac{|Z|^{2 n}}{n !},
$$

we deduce

$$
\left.P_{n}(t)\right|_{\phi=0}=\frac{2 \pi e^{-|Z|^{2}}|Z|^{2 n}}{n !}=\frac{2 \pi\left|\gamma \alpha_{1}(t)-i \zeta_{1}(t)\right|^{2 n} e^{-\left|\gamma \alpha_{1}(t)-i \zeta_{1}(t)\right|^{2}}}{n !},
$$

which is a Poisson distribution with the mean number parameter $\langle n\rangle$ given by

$$
\langle n\rangle=\left|\gamma \alpha_{1}(t)-i \zeta_{1}(t)\right|^{2} .
$$

\section{CONCLUSION}

We have considered a prototype Hamiltonian describing a driven, dissipative bosonic mode in a Kerr-like medium and found a generating function Eq. (18) to find the exact matrix elements of the reduced density matrix. The generating function is given in terms of the well-known normal characteristic functions in quantum Optics. For the case of a thermal environment in equilibrium with a Maxwell-Boltzmann state, an explicit expression for the components of the reduced density matrix is given by Eq. (23). Explicit expressions for reduced density matrix components are obtained for a bosonic mode initially prepared in a coherent state.

[1] H.-P. Breuer, F. Petruccione, The Theory of Open Quantum Systems (Oxford University Press, Oxford, 2002)

[2] A. Lampo, Quantum Brownian motion revisited: extensions and applications. Doctorial thesis, (Universitat Politècnica de Catalunya, Catalunya) (2018)

[3] U. Weiss, Quantum Dissipative Systems, 2nd edn. (World Scientific, Singapore, 1999)

[4] A.O. Caldeira, An Introduction to Macroscopic Quantum Phenomena and Quantum Dissipation (Cambridge University Press, Cambridge, 2014)

[5] J. Schwinger, J. Math. Phys. 2, 407 (1961)

[6] R.P. Feynman, F.L.J. Vernon, Ann. Phys. (N.Y.) 24, 118 (1963)

[7] A.O. Caldeira, A.J. Leggett, Physica A 121, 587 (1983) 
[8] H. Grabert, P. Schramm, G.-L. Ingold, Phys. Rep. 168, 115 (1988)

[9] M. Carlesso, A. Bassi, Phys. Rev. A 95, 052119 (2017)

[10] J. Gemmer, M. Michel, G. Mahler, Quantum Thermodynamics, 2nd edn. (Springer, Berlin, 2009)

[11] S. Gasparinetti, P. Solinas, A. Braggio, M. Sassetti, New J. Phys. 16, 115001 (2014)

[12] M. Carrega, P. Solinas, A. Braggio, M. Sassetti, U. Weiss, New J. Phys. 17, 045030 (2015)

[13] W. Dou, M.A. Ochoa, A. Nitzan, J.E. Subotnik, Phys. Rev. B 98, 134306 (2018)

[14] R.S. Whitney, Phys. Rev. B 98, 085415 (2018)

[15] K. Funo, H.T. Quan, Phys. Rev. Lett. 121, 040602 (2018)

[16] M. Perarnau-Llobet, H. Wilming, A. Riera, R. Gallego, J. Eisert, Phys. Rev. Lett. 120, 120602 (2018)

[17] M. A. Ochoa, N. Zimbovskaya, A. Nitzan, Phys. Rev. B 97, 085434 (2018)

[18] P. Haughian, M. Esposito, T.L. Schmidt, Phys. Rev. B 97, 085435 (2018)

[19] J. Lekscha, H. Wilming, J. Eisert, R. Gallego, Phys. Rev. E 97, 022142 (2018) 


\section{Supplementary Files}

This is a list of supplementary files associated with this preprint. Click to download.

- SupplementaryMat.pdf 